\title{
Comparison of computed tomography- and magnetic resonance imaging-based target delineation for cervical cancer brachytherapy
}

\author{
Fang Wang, MD!, Luyi Bu, MD!, Qun Wu, MD!, Xue Jiang, MD!' Lingyun Wu, MD, PhD!, Yu Li, MD!, Bin Xi, MD², \\ Zhongjie Lu, MD', Senxiang Yan, MD, PhD' \\ 'Department of Radiotherapy, First Affiliated Hospital, Zhejiang University School of Medicine, Hangzhou, China, ${ }^{2}$ Department of General \\ Surgery, First Affiliated Hospital, Zhejiang University School of Medicine, Hangzhou, China
}

\begin{abstract}
Purpose: The objective of this study was to compare and assess the accuracy of computed tomography (CT)-based target delineation with that of magnetic resonance imaging (MRI)-based on high-dose-rate brachytherapy (HDR-BT) for patients with cervical cancer.

Material and methods: Data of 20 patients with locally advanced cervical cancer were collected and evaluated. Dimensions, conformity, and dose parameters of high-risk clinical target volume $\left(\mathrm{CTV}_{\mathrm{HR}}\right)$ as well as $\mathrm{D}_{0.1 \mathrm{cc}} \mathrm{D}_{1 \mathrm{cc}}$ and $\mathrm{D}_{2 \mathrm{cc}}$ of organs at risk (OARs) based on MRI were compared with those based on CT.

Results: Average age of 20 patients included was 57.8 years. Width, thickness, and volumes of CT-based CTV $\mathrm{HR}_{\mathrm{HR}}$ $\left(\mathrm{CTV}_{\mathrm{HR}}-\mathrm{CT}\right)$ were significantly overestimated compared with those of MRI-based $\mathrm{CTV}_{\mathrm{HR}}\left(\mathrm{CTV}_{\mathrm{HR}}-\mathrm{MR}\right)$. Mean values of dice similarity coefficient (DSC), Hausdorff distance (HD), and centroid distance $(\Delta \mathrm{V})$ of $\mathrm{CTV}_{\mathrm{HR}}$ were $0.82 \mathrm{~cm}, 0.96 \mathrm{~cm}$, and $0.35 \mathrm{~cm}$, respectively. Dose values of $\mathrm{CTV}_{\mathrm{HR}}-\mathrm{CT}$ were significantly lower compared with those of $C T V_{\mathrm{HR}}-\mathrm{MR}$. Concerning OARs, geometrical and dosimetric values on CT were comparable to those on MRI.

Conclusions: The delineated ranges of $\mathrm{CTV}_{\mathrm{HR}}$ were significantly over-estimated on CT compared with MRI. $\mathrm{D}_{98}$ and $\mathrm{D}_{90}$ of $\mathrm{CTV}_{\mathrm{HR}}-\mathrm{CT}$ were lower than $\mathrm{CTV}_{\mathrm{HR}}-\mathrm{MR}$. DSC and $\Delta \mathrm{V}$ of $\mathrm{CTV}_{\mathrm{HR}}$ and $\mathrm{CTV}_{\mathrm{IR}}$ were similar to each other; however, there was a difference in terms of HD. CT images regarding pre-BT MR images for delineating were not enough and MRI fusion is still required.

J Contemp Brachytherapy 2020; 12, 4: 367-374 DOI: https://doi.org/10.5114/jcb.2020.98117
\end{abstract}

Key words: cervical cancer, BT, CT, MRI, conformity.

\section{Purpose}

Radiation therapy plays an important role in the treatment of cervical cancer. Intracavitary brachytherapy (BT) has shown to increase survival and local control [1] in addition to external beam radiation therapy (EBRT). Image-guided BT (IGBT) describes the incorporation of diagnostic radiology modalities into the process of $\mathrm{BT}$, such as applicator insertion, treatment planning, and treatment delivery. The increased use of 3-dimensional (3D) treatment planning for cervical cancer has helped us to further improve oncological outcomes and to reduce treatment-related toxicities.

Nowadays, computed tomography (CT) and magnetic resonance imaging (MRI) are widely used in 3D BT of cervical cancer. Radiation oncologists are very familiar with the interpretation of CT-based treatment planning, which provides useful volumetric information, such as the tandem position within the uterus, the thickness of recto-vaginal septum, and the relationship between bladder/recto-sigmoid and the applicator. It can also provide an accurate delineation of organs at risk (OARs) comparing with MRI [2] and allows for optimization of dose to OARs. Additionally, it maintains parameters traditionally used in the reporting system (e.g., point A) [3]. However, it is difficult for CT to accurately identify the target volume as it cannot clearly distinguish between structures of residual gross tumor volume (GTV), cervix, uterus, and vagina. Also, CT provides a poor definition of parametrial tumor infiltration [4]. Hence, contouring may be improved by incorporating information from MRI and clinical examination [5].

MRI is superior to CT for soft tissue visualization. It enables distinction of tumor from normal cervix and uterus, and visualization of cancerous infiltration into adjacent tissues (parametria, vagina) [6]. Accurate delineation of the tumor ensures that an adequate radiation dose is prescribed to the target, leading to excellent control rates. 
The Group European de Curiethérapie-European Society for Therapeutic Radiology and Oncology (GEC-ESTRO) recommended MRI for target delineation in cervical BT [7]. However, various survey reports demonstrated that there was a limited availability of MRI $[8,9]$. Also, accurate contouring with MRI requires appropriate training and experience. Special MRI-compatible BT applicators are considerably more expensive than metallic applicators. Most institutions in China do not have MRI available for BT planning yet. The purpose of this study was to investigate the volumetric and dosimetric difference between MRI- and CT-based BT in the treatment of cervical cancer.

\section{Material and methods}

Twenty patients with cervical cancer, who were treated with EBRT and five to six fractions of high-dose-rate (HDR) BT between January 2017 and April 2018 were included in the study. This study was approved by the institutional review board (IRB) of our hospital. Each patient underwent both CT (Sensation Open-24, Siemens, Forchheim, Germany) and MRI scan (Optima MR360 1.5 T, GE Medical Systems Inc., Waukesha, WI, USA) for the first fraction of BT plan. The two scans were acquired with- in one hour, with the applicator (Henschke tandem, left ovoid, right ovoid) fixed to the target. This was performed through vaginal packing and the use of bladder filling and rectum preparation protocols [10]. MRI without the applicator before BT (pre-BT MRI) was also collected to be used for contouring on CT images. Note that it was used for reference only, not fused into the CT image at BT. T2-weighted sequences in the axial, coronal, and sagittal planes were acquired at $5 \mathrm{~mm}$ slice thickness during the MRI scan. In the next applications, CT planning was performed. The CT images for BT were generated in a $3 \mathrm{~mm}$ slice interval without intravenous contrast.

Gynecological examinations were performed before EBRT and BT, which provided the reference for subsequent target delineation and planning. The image data sets were contoured using Varian system (Eclipse 11.0) by the same radiation oncologist. The volume of high-risk clinical target volume $\left(\mathrm{CTV}_{\mathrm{HR}}\right)$ and intermediate-risk CTV $\left(\mathrm{CTV}_{\mathrm{IR}}\right)$ of MRI were identified according to GEC-ESTRO recommendations [7], while the adapted guidelines by Viswanathan et al. [11] were used for CTbased contouring. CT images were contoured before MRI. Bladder, rectum, sigmoid colon, and small intestine were also contoured. Figure 1 shows $\mathrm{CTV}_{\mathrm{HR}}$ and $\mathrm{CTV}_{\mathrm{IR}}$ both on CT and MRI of the same patient. Rigid registration

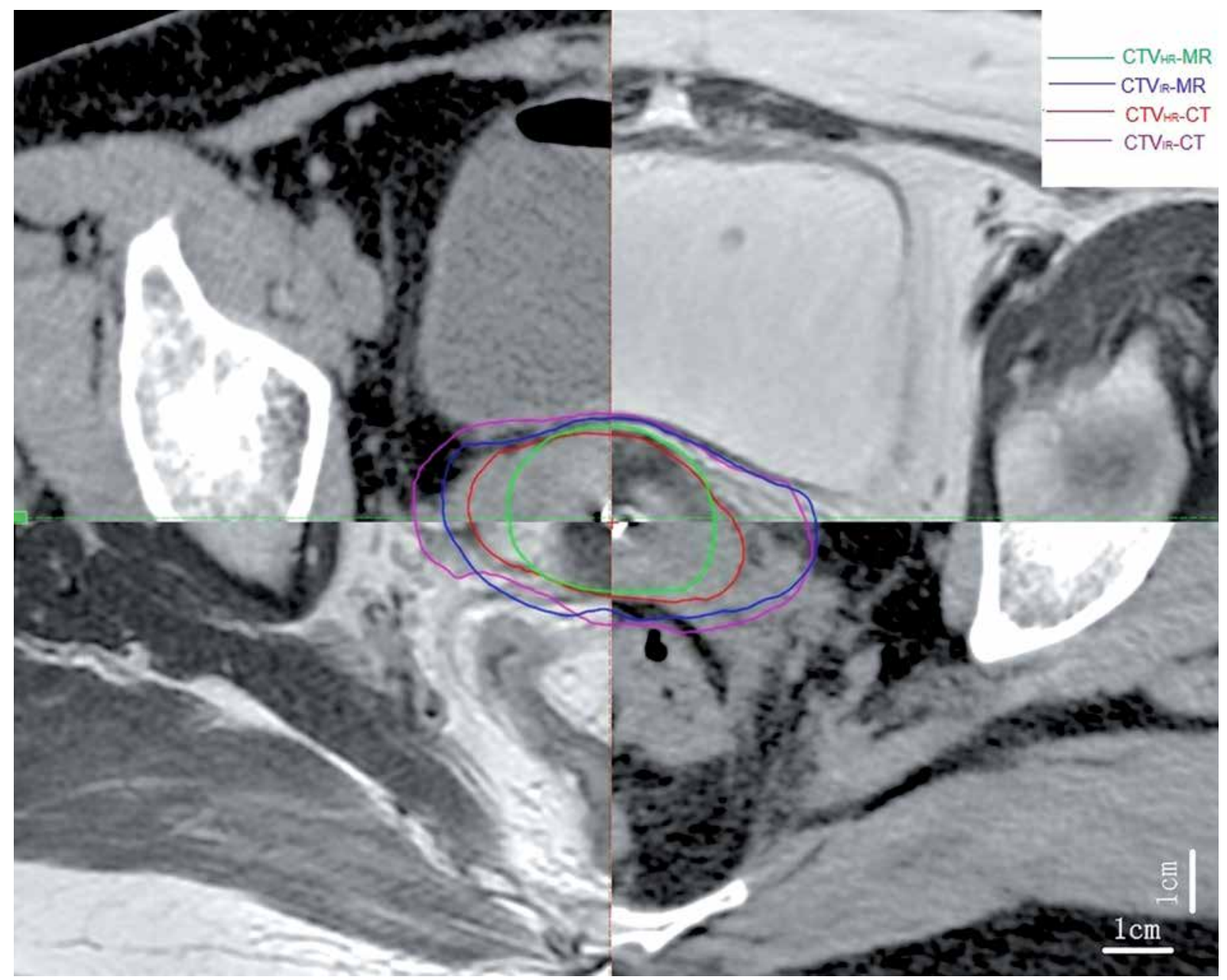

Fig. 1. $C T V_{\mathrm{HR}}$ and $C T V_{I R}$ of both $\mathrm{CT}$ and MRI for a 53-year-old woman with stage IIB cervical cancer 
Table 1. The dimensions and volumes of $\mathrm{CTV}_{\mathrm{HR}}$ from $\mathrm{CT}$ and $\mathrm{MRI}$

\begin{tabular}{llll} 
Dimension & $\mathrm{CTV}_{\mathrm{HR}^{-}}-\mathrm{CT}$ & $\mathrm{CTV}_{\mathrm{HR}^{-}}-\mathrm{MR}$ & $P$-value \\
\hline Maximum width $(\mathrm{cm})$ & $4.47 \pm 0.97$ & $4.20 \pm 1.12$ & 0.120 \\
\hline Width at point A $(\mathrm{cm})$ & $3.42 \pm 0.82$ & $3.02 \pm 0.67$ & 0.018 \\
\hline Maximum thickness $(\mathrm{cm})$ & $3.19 \pm 0.61$ & $2.97 \pm 0.56$ & 0.001 \\
\hline Thickness at point A $(\mathrm{cm})$ & $2.93 \pm 0.57$ & $2.73 \pm 0.63$ & 0.013 \\
\hline Height $(\mathrm{cm})$ & $4.54 \pm 1.45$ & $4.82 \pm 1.44$ & 0.095 \\
\hline Volume $(\mathrm{cc})$ & $44.50 \pm 11.35$ & $39.24 \pm 11.7$ & 0.006
\end{tabular}

results of MRI-CT images were obtained using MIM software registration tool (Maestro, version 6.7.4) to fuse the structures of the applicator on the two images. Treatment planning was done on the Varian treatment planning system (Varian planning system, Eclipse 11.0), where the DVH parameters were calculated on CT. Then, the same planning was transferred to MRI with the values recorded. Five to six sessions of BT application were planned for all patients, with a fraction size of 5-6 Gy to $\mathrm{D}_{90}$ of $\mathrm{CTV}_{\mathrm{HR}}$ per session, according to the GEC-ESTRO recommendations [7]. The total doses of EBRT and BT were summated and normalized to a 2 Gy fraction size $\left(\mathrm{EQD}_{2}\right)$, using the linear-quadratic model with an $\alpha / \beta$ of 3 Gy for OARs and $10 \mathrm{~Gy}$ for $\mathrm{CTV}_{\mathrm{HR}}$ [12]. The planned $\mathrm{EQD}_{2} \mathrm{D}_{90}$ for $\mathrm{CTV}_{\mathrm{HR}}$ was $\geq 85 \mathrm{~Gy}$, while the doses to $2 \mathrm{cc}$ volumes of rectum, bladder, and sigmoid were limited to $\leq 75, \leq 85$, and $\leq 75 \mathrm{~Gy}$, respectively.

The dimensions, dice similarity coefficient (DSC, the overlapping parts between two target areas), Hausdorff distance (HD, the relative distance between the two points of greatest difference), centroid distance $(\Delta \mathrm{V}$, the deviation degree of central points of two target areas), and dose volume histograms (DVH) parameters were analyzed.

We used the MIM system to calculate the values of DSC, HD, and DV. Doses to $0.1 \mathrm{cc}, 1 \mathrm{cc}$, and $2 \mathrm{cc}$ volumes of the OARs of MRI and CT were evaluated. All MRI parameters were compared with those of CT by paired-samples t-test using SPSS version 23.0 software. The significance test was set to the level of $p<0.05$.

\section{Results}

\section{Patients and treatment characteristics}

The average age of the 20 patients included was 57.8 years (range, 41-75 years old). Pathologically, all the patients were of squamous cell carcinoma, including 1 with stage IB2, 1 with stage IIA2, 13 patients with stage IIB, 3 with stage IIIA, and 2 cases with stage IIIB disease with both pelvic wall infiltration and hydronephrosis. A total dose of 45-50.4 Gy in 25-28 fractions was prescribed using 6-10 MV photons for EBRT. Tumor reduction exceeding $50 \%$ or $1 \mathrm{~cm}$ was observed in 19 patients at the time of BT. The first BT application was carried out in $4^{\text {th }}$ week of EBRT for 15 patients, while the other 5 patients received the treatment after the completion of EBRT. The treatment was completed within 56 days for all the patients.

\section{Comparison of dimensions and target volumes}

The mean values with standard deviation for the height, maximum width, width at point $\mathrm{A}$, maximum thickness, thickness at point $\mathrm{A}$, and volumes of $\mathrm{CTV}_{\mathrm{HR}}-\mathrm{CT}$ and $\mathrm{CTV}_{\mathrm{HR}} \mathrm{MR}$ are presented in Table 1. Two-sided paired $t$-tests showed that the width at point $\mathrm{A}$, maximum thickness, thickness at point $\mathrm{A}$, and total volumes were significantly overestimated in $\mathrm{CTV}_{\mathrm{HR}}-\mathrm{CT}$ compared with $\mathrm{CTV}_{\mathrm{HR}}-\mathrm{MR}$. The height of $\mathrm{CTV}_{\mathrm{HR}}-\mathrm{CT}$ was comparable to $\mathrm{CTV}_{\mathrm{HR}}-\mathrm{MR}$.

\section{Comparison of conformal parameters of DSC, HD, and $\Delta V$}

The mean values and the range of DSC, $\mathrm{HD}$, and $\Delta \mathrm{V}$ of $\mathrm{CTV}_{\mathrm{HR}}$ and $\mathrm{CTV}_{\mathrm{IR}}$ are shown in Table 2 and Figure 2. Conformality indices of both $\mathrm{CTV}_{\mathrm{HR}}$ and $\mathrm{CTV}_{\mathrm{IR}}$ were comparable.

\section{Comparison of dose parameters}

The $D_{98}$ and $D_{90}$ values were significantly lower for $\mathrm{CTV}_{\mathrm{HR}}-\mathrm{CT}$ as compared to $\mathrm{CTV}_{\mathrm{HR}}-\mathrm{MR}$ (Table 3).

The contours of the bladder, rectum, sigmoid, and small intestine appeared to properly overlap on both CT and MRI scans (Figure 3). No statistical difference was observed for the doses to $0.1 \mathrm{cc}, 1 \mathrm{cc}$, and $2 \mathrm{cc}$ volumes for the above OARs between CT and MRI (Table 4).

\section{Discussion}

For more than a decade, the application of 3D imaging both during and after BT insertion has increased globally $[13,14]$. A 3D imaging-based BT has been well recognized as a much more precise technique than the traditional orthogonal radiographs. It allows for optimal dose coverage of the targets, leading to improvements in

Table 2. The conformal parameters of $\mathrm{CTV}_{\mathrm{HR}}$ and $\mathrm{CTV}_{\mathrm{IR}}$ from $\mathrm{CT}$ and MRI

\begin{tabular}{lcc} 
Conformity & CTV $_{\mathrm{HR}}$ & CTV $_{\mathrm{IR}}$ \\
\hline $\mathrm{DSC}$ & $0.82(0.76-0.89)$ & $0.79(0.74-0.91)$ \\
\hline $\mathrm{HD}(\mathrm{cm})$ & $0.96(0.65-1.35)$ & $0.99(0.55-1.42)$ \\
\hline$\Delta \mathrm{V}(\mathrm{cm})$ & $0.35(0.15-0.56)$ & $0.33(0.16-0.56)$
\end{tabular}




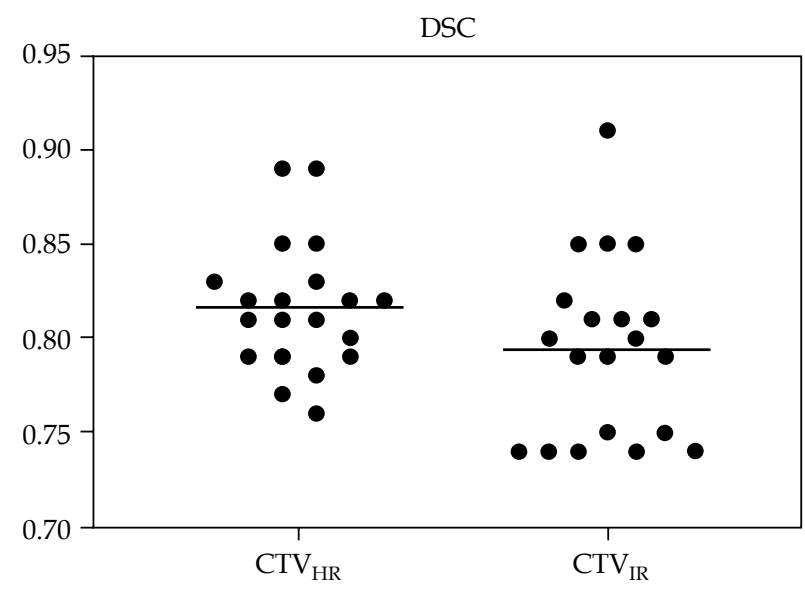

$\Delta \mathrm{V}(\mathrm{cm})$

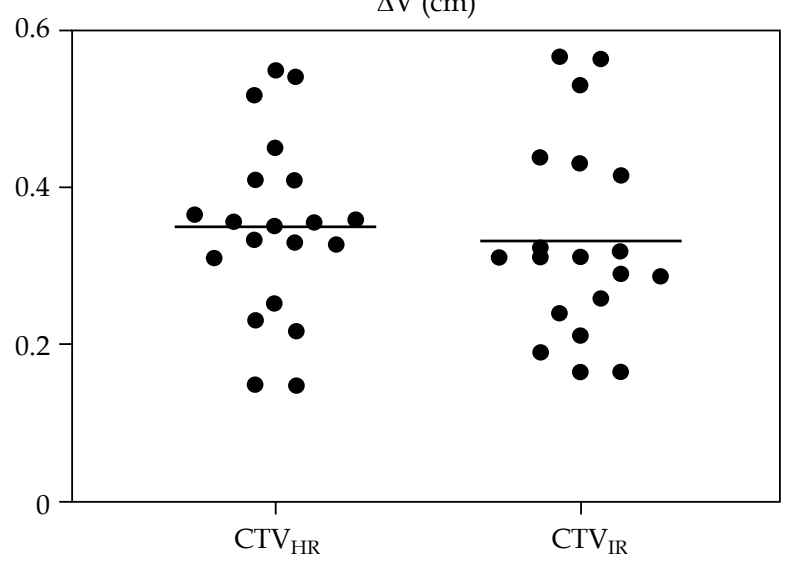

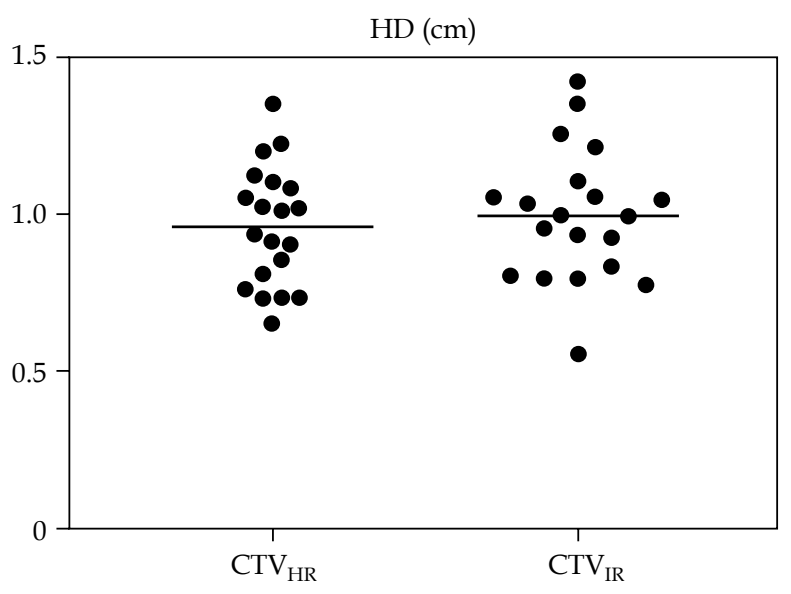

Fig. 2. Chart of the conformal parameters of DSC, HD, and $\Delta \mathrm{V}$ for each patient

Table 3. DVH parameters of $C T V_{H R}$ and $C T V_{I R}$ from $C T$ and $M R I$

\begin{tabular}{lllcccc} 
Parameters & $\mathrm{CTV}_{\mathrm{HR}}-\mathrm{CT}$ & $\mathrm{CTV}_{\mathrm{HR}^{-} \mathrm{MR}}$ & $P$-value & $\mathrm{CTV}_{\mathrm{IR}^{-}} \mathrm{CT}$ & $\mathrm{CTV}_{\mathrm{IR}^{-}}-\mathrm{MR}$ & $P$-value \\
\hline $\mathrm{D}_{98}$ (Gy) & $4.43 \pm 0.51$ & $4.82 \pm 0.54$ & 0.030 & $2.50 \pm 0.38$ & $2.67 \pm 0.38$ & 0.269 \\
\hline $\mathrm{D}_{90}$ (Gy) & $5.23 \pm 0.68$ & $5.63 \pm 0.62$ & 0.035 & $3.13 \pm 0.35$ & $3.28 \pm 0.51$ & 0.219
\end{tabular}

oncological outcomes [15]. CT images are by far the most used data for 3D BT. There are only a few institutions that own dedicated MRI for direct BT planning. However, the limitations of delineated targets on CT images were noted when compared to MRI-based ones [11,16,17,18]. Herein, our study assessed the accuracy of CT versus MRI, utilizing conformality indices, geometrical dimensions, and dose parameters.

In our study, the target dimensions, width, and thickness were significantly overestimated in CT compared with MRI, while the height was comparable. Because of the overestimated width and thickness, the volumes were significantly larger in $\mathrm{CTV}_{\mathrm{HR}}-\mathrm{CT}$. These differences come from the improved visibility of T2-weighted MRI. It can distinguish and better delineate soft tissue and tumor in the pelvis. The parametric ligaments were depicted with a wide variation in shape and thickness on CT [19]. Therefore, wide $\mathrm{CTV}_{\mathrm{HR}}$ volumes may be outlined on CT images for patients with a uterosacral or parametrial extension [2]. Several studies indicated that the height was similar between CT and MRI [11,20,21]. Rai et al. [22] reported that the height was significantly under- estimated in $\mathrm{CTV}_{\mathrm{HR}}-\mathrm{CT}$ contours; however, the statistics were similar between the heights of $\mathrm{CT}+\mathrm{MRI}$ at diagnosis $\left(\mathrm{CTV}_{\mathrm{HR}}-\mathrm{CT}_{\mathrm{dMRI}}\right)$ and $\mathrm{CT}+\mathrm{MRI}$ at $\mathrm{BT}\left(\mathrm{CTV}_{\mathrm{HR}}-\mathrm{MR}\right)$. Viswanathan et al. [11] considered that CT contouring results can be improved by contrast-enhanced imaging and careful integration of information obtained from clinical examination and MRI immediately before BT. Although the tumor itself generally does not exhibit strong enhancement post-EBRT, uterine vessels can be identified as surrogates in the delineation of cervical-uterine junction on contrast-enhanced CT images. This junction can guide contouring of the superior border of $\mathrm{CTV}_{\mathrm{HR}}$. Mahantshetty et al. [23] suggested that a CT-based target using MRI at diagnosis and real-time transrectal ultrasonography data during BT seems comparable to the gold standard of MRI-based approach in IGABT for cervical cancer. In our study, pre-BT MRI and clinical examination were performed and used as references for contouring on CT images. The tumors were significantly reduced after EBRT and heights of tumors shrunk to the cervix in 12 cases before BT. Therefore, there was no significant difference in the height between MRI and CT in this study. 


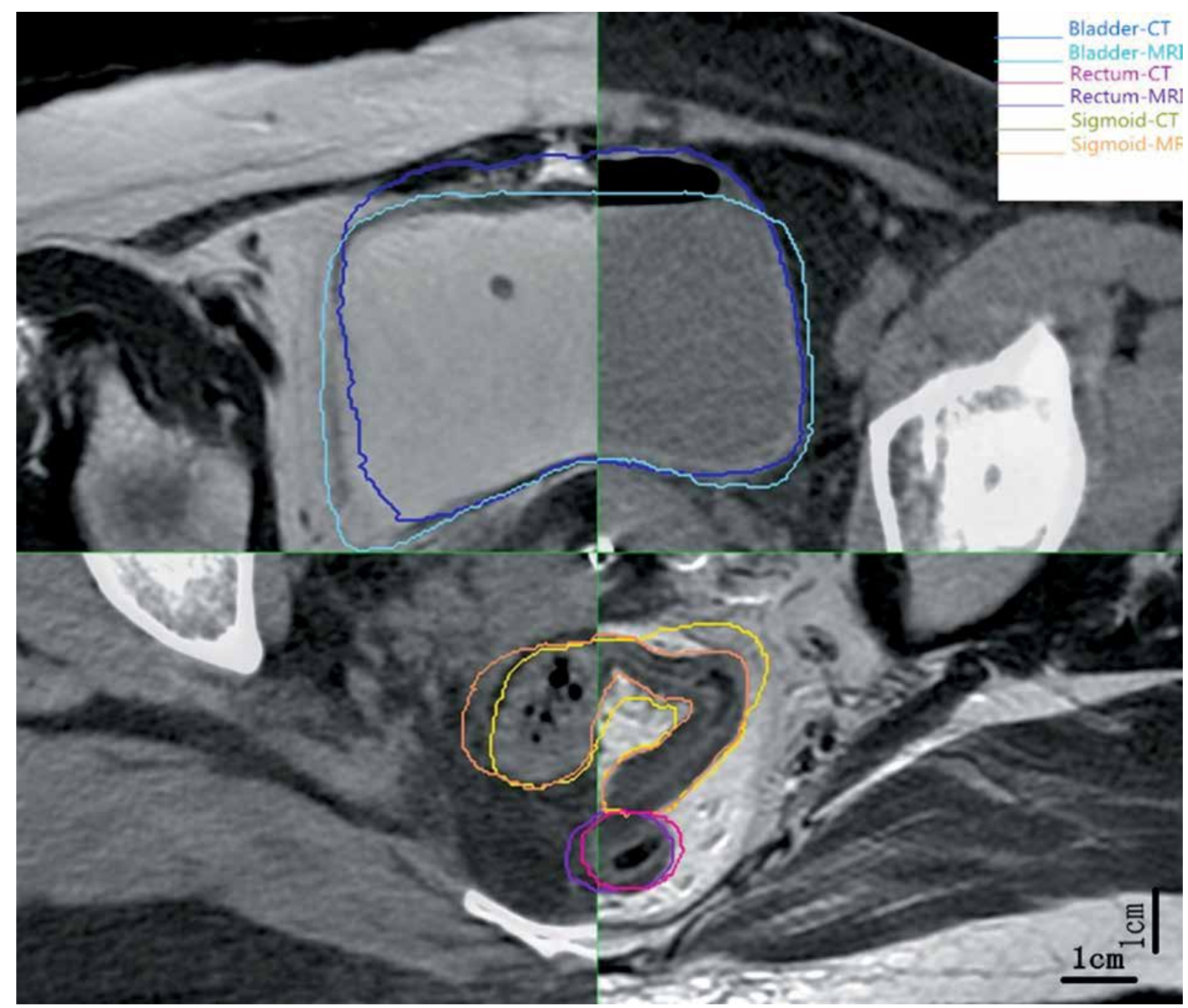

Fig. 3. The contours of OARs on both CT and MRI scans

Table 4. Mean doses with standard deviation for OARs with CT and MRI

\begin{tabular}{|c|c|c|c|c|}
\hline OARs doses (Gy) & $\mathrm{CT}$ & MRI & Total EQD 2 & $P$-value \\
\hline \multicolumn{5}{|l|}{ Bladder } \\
\hline $\mathrm{D}_{0.1 c c}$ & $6.09 \pm 0.57$ & $6.09 \pm 0.60$ & $97.50 \pm 5.79$ & 1.000 \\
\hline $\mathrm{D}_{1 \mathrm{cc}}$ & $5.13 \pm 0.48$ & $5.41 \pm 0.52$ & $85.55 \pm 3.94$ & 0.052 \\
\hline $\mathrm{D}_{2 \mathrm{cc}}$ & $4.79 \pm 0.42$ & $4.90 \pm 0.41$ & $81.15 \pm 3.67$ & 0.375 \\
\hline \multicolumn{5}{|l|}{ Rectum } \\
\hline $\mathrm{D}_{0.1 \mathrm{ccc}}$ & $4.87 \pm 1.07$ & $5.03 \pm 1.25$ & $80.27 \pm 10.33$ & 0.265 \\
\hline $\mathrm{D}_{1 \mathrm{cc}}$ & $3.85 \pm 0.86$ & $4.10 \pm 1.06$ & $70.27 \pm 9.39$ & 0.186 \\
\hline$D_{2 c c}$ & $3.45 \pm 0.77$ & $3.77 \pm 1.11$ & $65.95 \pm 7.67$ & 0.074 \\
\hline \multicolumn{5}{|l|}{ Sigmoid } \\
\hline$\underline{\mathrm{D}_{0.1 \mathrm{cc}}}$ & $3.85 \pm 1.22$ & $4.16 \pm 1.78$ & $68.65 \pm 8.47$ & 0.389 \\
\hline$D_{1 c c}$ & $3.06 \pm 1.02$ & $3.28 \pm 1.28$ & $63.25 \pm 8.40$ & 0.315 \\
\hline$D_{2 c c}$ & $2.75 \pm 0.95$ & $2.94 \pm 1.15$ & $60.29 \pm 7.44$ & 0.302 \\
\hline \multicolumn{5}{|l|}{ Small intestine } \\
\hline $\mathrm{D}_{0.1 \mathrm{cc}}$ & $3.98 \pm 1.41$ & $3.72 \pm 1.46$ & $48.68 \pm 2.45$ & 0.055 \\
\hline$D_{1 c c}$ & $2.95 \pm 1.01$ & $2.70 \pm 0.88$ & $46.83 \pm 3.34$ & 0.088 \\
\hline$D_{2 c c}$ & $2.75 \pm 0.97$ & $2.44 \pm 0.95$ & $44.57 \pm 2.57$ & 0.070 \\
\hline
\end{tabular}


CT images were comparable to MRI for contouring OARs in our study. The difference in the volume of rectum was occasionally statistically significant because of the interval time between MRI and CT [24].

The parameters of volume, length, width, and height can be accounted for the general shape of CTV comparing CT with MRI, while the DSC, HD, and $\Delta \mathrm{V}$ can shed light on the spatial correlation between two groups [25]. These parameters have been commonly used in a comparative study of target areas and to evaluate the accuracy of 3D medical image segmentation [26]. Pötter et al. [18] analyzed conformity index $(\mathrm{CI})$ between $\mathrm{CT}$ contours and reference contours on MRI with the applicator in place. They found that most disagreements occurred between the contour set in caudal and cranial regions of $\mathrm{CTV}_{\mathrm{HR}}-\mathrm{MR}$. The $\mathrm{CI}$ and coefficient of variation $(\mathrm{CV})$ of OARs volumes contoured across physicians for patients treated with interstitial brachytherapy had been reported by Damato et al. [27]. Twenty-three gynecologic radiation oncology experts contoured the same 3 cervical cancer BT patients to compare CT with MRI contours, and found that Dice coefficients were $89 \%$ for stage IB2 patient with complete response (CR), $74 \%$ for stage IIB case with partial response (PR), and 57\% for stage IIB patient with CR [28]. Mason et al. compared $\mathrm{CTV}_{\mathrm{HR}}$ with corresponding cone beam CT (CBCT), ultrasound (US), and CBCT-US-fused images using DSC (range, 0.81-0.84) [29]. Novakova et al. [30] used $\mathrm{HD}$ and $\Delta \mathrm{V}$ to determine the optimal number of plans for locally advanced cervical cancer and found HD to be an easy-to-implement criterion to select those patients' pre-treatment. HD was used to compare differences in the contour edge, while $\Delta \mathrm{V}$ presented differences in the inner center of a mass. These three parameters can comprehensively compare the internal and external shape differences between the two target areas. On the other hand, the conformity parameters can also provide a preliminary evaluation of the fusion quality of CT and MR images. DSC above 0.70 presented good result [31]. The values of $\mathrm{HD}$ and $\Delta \mathrm{V}$ parameters below $0.5 \mathrm{~cm}$ showed little difference, and the smaller their values, the better the results [32]. The differences in conformal parameters of DSC, $\mathrm{HD}$, and $\Delta \mathrm{V}$ between the two target areas in our study were mainly due to the following reasons: 1 . The differences in the display of soft tissues between MRI and CT; 2. The differences in the slice thickness of MRI and CT, which was $5 \mathrm{~mm}$ for MRI and $3 \mathrm{~mm}$ for CT, and could impact the delineation of the target area; 3 . Each patient underwent CT first and then MRI scan. The CT scan process only needs no more than 1 minute, while the MRI scan needs about 15 minutes. Therefore, there was a time gap between the two scans. The bladder size and intestinal filling change during the MRI scanning, which might lead to differences in boundaries of $\mathrm{CTV}_{\mathrm{IR}}$. According to our study, DSC and $\triangle \mathrm{V}$ of $\mathrm{CTV}_{\mathrm{HR}}$ and $\mathrm{CTV}_{\mathrm{IR}}$ comparing $\mathrm{CT}$ with MRI were similar; however, the difference in HD values was relatively large. CT images regarding pre-BT MR images for delineating were still not enough.

For adaptive planning of cervix cancer BT, ABS guidelines recommended that $3 \mathrm{D}$ imaging with ultrasound, CT, or MRI should be performed when feasible to estimate cervical tumor dimensions and ensure adequate coverage of the tumor [33]. In our study, the $\mathrm{D}_{90}$ and $\mathrm{D}_{98}$ for the $\mathrm{CTV}_{\mathrm{HR}}$ and $\mathrm{CTV}_{\mathrm{IR}}$ with CT were compared with MRI. Because of the larger volume in $\mathrm{CTV}_{\mathrm{HR}}-\mathrm{CT}$, the $D_{90}$ and $D_{98}$ values were significantly lower. Since MRI is not available for planning of each BT fraction in all institutions where image-guided cervix cancer BT is performed, studies combining MRI and CT for different fractions have been reported. Beriwal et al. $[34,35]$ were first to report the use of MRI at the time of first BT planning with the applicator in place, followed by subsequent CT-based fractions comparing with single MRI for all fractions. Mean $\mathrm{D}_{90}$ for $\mathrm{CTV}_{\mathrm{HR}}$ and doses of OARs with the MRI-only were comparable to the MRI-CT method. Nesvacil et al. [36] described a technique of combining MRI for first BT fraction and CT for subsequent fractions. The results showed a striking similarity between cases of small tumors and intracavitary applications with fully MRI-based planning, both in terms of $\mathrm{CTV}_{\mathrm{HR}}$ coverage and corresponding OARs dose limits. For larger tumors, complicated applications, and situations with unfavorable OARs topography, MRI-based adaptive BT planning remains superior over CT-based. Choong et al. [8] reported their experiences of using an alternative CT/MRIbased (hybrid) approach, with all patients having a preBT MRI scan at 4 days before treatment and a second MRI scan with applicators in place at the first fraction. CT was used for subsequent fractions; the $\mathrm{CTV}_{\mathrm{HR}}$ from MRI at the first fraction was transferred into subsequent fraction CT image sets for the hybrid approach. Dosimetry and late toxicity rates achieved were similar between the two groups.

According to dose parameters of OARs, no significant differences between CT and MRI were identified in our study; CT images were comparable to MRI for contouring OARs. However, many studies have found improved dose avoidance using MRI approaches $[8,24,36]$. These results were obtained probably due to better identification of tumor delineation, the wall of bladder, rectum, and sigmoid, and the interval time between MRI and CT scans.

In future, we are planning to conduct more analysis on how to precisely fuse a part of pre-BT MR images with CT at BT to delineate the CTV area more accurately.

\section{Conclusions}

According to our study, the width and thickness of $\mathrm{CTV}_{\mathrm{HR}}$ are significantly overestimated on CT as compared to MRI. DSC and $\Delta \mathrm{V}$ of $\mathrm{CTV}_{\mathrm{HR}}$ and $\mathrm{CTV}_{\mathrm{IR}}$ are similar; however, there is a difference in HD. Dosimetrically, the $\mathrm{D}_{98}$ and $\mathrm{D}_{90}$ for $\mathrm{CTV}_{\mathrm{HR}}$ are lower for CT-based technique. Concerning OARs, CT images are comparable to MRI. These differences are due to the soft tissue resolution between CT and MRI, not the difference in organ geometry. CT images regarding pre-BT MR images for delineating are still not enough and MRI fusion is required.

\section{Acknowledgments}

This study was supported by the following grants: Zhejiang Medical and Health Science and Technology 
Project, 2018258853, and Zhejiang Provincial Natural Science Foundation, LQ19H160032.

\section{Disclosure}

The authors report no conflict of interest.

\section{References}

1. Lanciano RM, Won M, Coia LR et al. Pretreatment and treatment factors associated with improved outcome in squamous cell carcinoma of the uterine cervix: a final report of the 1973 and 1978 patterns of care studies. Int J Radiat Oncol Biol Phys 1991; 20: 667-676.

2. Bipat S, Glas AS, van der Velden J et al. Computed tomography and magnetic resonance imaging in staging of uterine cervical carcinoma: a systematic review. Gynecol Oncol 2003 91: 59-66.

3. Datta NR, Srivastava A, Maria Das KJ et al. Comparative assessment of doses to tumor, rectum, and bladder as evaluated by orthogonal radiographs vs. computer enhanced computed tomography-based intracavitary brachytherapy in cervical cancer. Brachytherapy 2006; 5: 223-229.

4. Mitchell DG, Snyder B, Coakley F et al. Early invasive cervical cancer: tumor delineation by magnetic resonance imaging, computed tomography, and clinical examination, verified by pathologic results, in the ACRIN 6651/GOG 183 Intergroup Study. J Clin Oncol 2006; 24: 5687-5694.

5. Hegazy N, Potter R, Kirisits C et al. High-risk clinical target volume delineation in CT-guided cervical cancer brachytherapy: impact of information from FIGO stage with or without systematic inclusion of 3D documentation of clinical gynecological examination. Acta Oncol 2013; 52: 1345-1352.

6. Nag S. Controversies and new developments in gynecologic brachytherapy: image-based intracavitary brachytherapy for cervical carcinoma. Semin Radiat Oncol 2006; 16: 164-167.

7. Haie-Meder C, Potter R, Van Limbergen E et al. Recommendations from Gynaecological (GYN) GEC-ESTRO Working Group (I): concepts and terms in 3D image based 3D treatment planning in cervix cancer brachytherapy with emphasis on MRI assessment of GTV and CTV. Radiother Oncol 2005; 74: 235-245.

8. Choong ES, Bownes P, Musunuru HB et al. Hybrid (CT/ MRI based) vs. MRI only based image-guided brachytherapy in cervical cancer: Dosimetry comparisons and clinical outcome. Brachytherapy 2016; 15: 40-48.

9. Wang F, Tang Q, Lv G et al. Comparison of computed tomography and magnetic resonance imaging in cervical cancer brachytherapy: A systematic review. Brachytherapy 2017; 16: 353-365.

10. Nesvacil N, Tanderup K, Hellebust TP et al. A multicentre comparison of the dosimetric impact of inter- and intra-fractional anatomical variations in fractionated cervix cancer brachytherapy. Radiother Oncol 2013; 107: 20-25.

11. Viswanathan AN, Dimopoulos J, Kirisits C et al. Computed tomography versus magnetic resonance imaging-based contouring in cervical cancer brachytherapy: results of a prospective trial and preliminary guidelines for standardized contours. Int J Radiat Oncol Biol Phys 2007; 68: 491-498.

12. Kirisits C, Potter R, Lang S et al. Dose and volume parameters for MRI-based treatment planning in intracavitary brachytherapy for cervical cancer. Int J Radiat Oncol Biol Phys 2005; 62: 901-911.

13. Viswanathan AN, Erickson BA. Three-dimensional imaging in gynecologic brachytherapy: a survey of the American Brachytherapy Society. Int J Radiat Oncol Biol Phys 2010; 76: 104-109.
14. Viswanathan AN, Creutzberg CL, Craighead P et al. International brachytherapy practice patterns: a survey of the Gynecologic Cancer Intergroup (GCIG). Int J Radiat Oncol Biol Phys 2012; 82: 250-255.

15. Potter R, Dimopoulos J, Georg P et al. Clinical impact of MRI assisted dose volume adaptation and dose escalation in brachytherapy of locally advanced cervix cancer. Radiother Oncol 2007; 83: 148-155.

16. Hricak H, Gatsonis C, Coakley FV et al. Early invasive cervical cancer: $\mathrm{CT}$ and MR imaging in preoperative evaluation ACRIN/GOG comparative study of diagnostic performance and interobserver variability. Radiology 2007; 245: 491-498.

17. Tuntipumiamorn L, Lohasammakul S, Dankulchai $\mathrm{P}$ et al. Comparison of impact of target delineation of computed tomography- and magnetic resonance imaging-guided brachytherapy on dose distribution in cervical cancer. J Contemp Brachytherapy 2018; 10: 418-424.

18. Potter R, Federico M, Sturdza A et al. Value of magnetic resonance imaging without or with applicator in place for target definition in cervix cancer brachytherapy. Int J Radiat Oncol Biol Phys 2016; 94: 588-597.

19. Foshager MC, Walsh JW. CT anatomy of the female pelvis: a second look. Radiographics 1994; 14: 51-64; discussion 64-56.

20. Federico M, Kholosy N, Nesvacil N et al. Pre-implant clinical and radiological MRI assessment improves CT-based HRCTV delineation in cervical cancer patients. Brachytherapy 2011; 10: S14-15.

21. Khadanga C, Mahantshetty U, Engineer S et al. Critical evaluation and comparison of CT vs MR based HRCTV and OAR contouring during IGBT for cervical cancer. Radiother Oncol 2015; 115: S141-142.

22. Rai B, Patel FD, Tomar P et al. A study to assess the feasibility of using CT ( \pm diagnostic MRI) instead of MRI at brachytherapy in image guided brachytherapy in cervical cancer. J Radiother Pract 2013; 13: S438-446.

23. Mahantshetty U, Naga CP, Khadanga CR et al. A prospective comparison of computed tomography with transrectal ultrasonography assistance and magnetic resonance imaging based target volume definition during image guided adaptive brachytherapy for cervical cancers. Int J Radiat Oncol Biol Phys 2018; 102: 1448-1456.

24. Tharavichitkul E, Samorn N, Wanwilairat S et al. The comparison of high-risk clinical target volumes between computed tomography and magnetic resonance imaging in cervical cancer. Biomed Imaging Interv J 2013; 9: 1-5.

25. Hague C, Beasley W, Dixon L et al. Use of a novel atlas for muscles of mastication to reduce inter observer variability in head and neck radiotherapy contouring. Radiother Oncol 2019; 130: 56-61.

26. Raudaschl PF, Zaffino P, Sharp GC et al. Evaluation of segmentation methods on head and neck CT: Auto-segmentation challenge 2015. Med Phys 2017; 44: 2020-2036.

27. Damato AL, Townamchai K, Albert M et al. Dosimetric consequences of interobserver variability in delineating the organs at risk in gynecologic interstitial brachytherapy. Int J Radiat Oncol Biol Phys 2014; 89: 674-681.

28. Viswanathan AN, Erickson B, Gaffney DK et al. Comparison and consensus guidelines for delineation of clinical target volume for CT- and MR-based brachytherapy in locally advanced cervical cancer. Int J Radiat Oncol Biol Phys 2014; 90: 320-328.

29. Mason SA, White IM, O'Shea T et al. Combined ultrasound and cone beam CT improves target segmentation for image guided radiation therapy in uterine cervix cancer. Int J Radiat Oncol Biol Phys 2019; 104: 685-693.

30. Novakova E, Heijkoop ST, Quint S et al. What is the optimal number of library plans in ART for locally advanced cervical cancer? Radiother Oncol 2017; 125: 470-477. 
31. Artaechevarria X, Munoz-Barrutia A, Ortiz-de-Solorzano C. Combination strategies in multi-atlas image segmentation: application to brain MR data. IEEE Trans Med Imaging 2009; 28: 1266-1277.

32. Men K, Dai J, Li Y. Automatic segmentation of the clinical target volume and organs at risk in the planning CT for rectal cancer using deep dilated convolutional neural networks. Med Phys 2017; 44: 6377-6389.

33. Viswanathan AN, Beriwal S, De Los Santos JF et al. American Brachytherapy Society consensus guidelines for locally advanced carcinoma of the cervix. Part II: high-dose-rate brachytherapy. Brachytherapy 2012; 11: 47-52.

34. Beriwal S, Kim H, Coon D et al. Single magnetic resonance imaging vs magnetic resonance imaging/computed tomography planning in cervical cancer brachytherapy. Clin Oncol (R Coll Radiol) 2009; 21: 483-487.

35. Beriwal S, Kannan N, Kim H et al. Three-dimensional high dose rate intracavitary image-guided brachytherapy for the treatment of cervical cancer using a hybrid magnetic resonance imaging/computed tomography approach: feasibility and early results. Clin Oncol (R Coll Radiol) 2011; 23: 685-690.

36. Nesvacil N, Potter R, Sturdza A et al. Adaptive image guided brachytherapy for cervical cancer: a combined MRI-/ CT-planning technique with MRI only at first fraction. Radiother Oncol 2013; 107: 75-81. 